margin. If this impression is subsequently confirmed it may raise our awareness of the problem and allow early, and hence more conservative, surgery on patients from south Asia.

ALAN YOUNG

Postbox 126,

Kathmandu,

Nepal

\section{Neonatal screening for haemoglobinopathies}

The article by Dr Paul D Griffiths and others (4 June, p 1583) prompts us to report our experience with neonatal screening of cord blood for abnormal haemoglobins.

Since 1978 we have screened children whose mothers are known to carry abnormal haemoglobins. According to the 1981 census, $10 \%$ of families in Reading have a head of the household who comes from the new Commonwealth. There are 6000 babies delivered in the main maternity unit each year, so at least 600 babies could be screened. Identification of those we need to screen has reduced our workload.

During the 10 years we have identified eight children with major haemoglobin disorders (two with $\mathrm{HbSS}$, one with $\mathrm{HbSC}$, and one with thalassaemia major). Most were born to couples known to be at risk. Two children with HbSS were identified where the mother was the only paren tested, and in these families most of the antenatal care had been done abroad. Two children with HbSB and thalassaemia have been lost to follow up. During this time three children in Reading have been diagnosed as suffering from phenylketonuria using Guthrie tests.

We missed one child with HbSC, from whom cord blood was not sent as the mother had had chorionic villus sampling elsewhere and had been told that her child had sickle cell trait. The referra centre was not told that $\mathrm{HbC}$ trait was also in the family. A four year old child with HbSS and splenic sequestration died. This child was born in Nigeria of mixed Anglo-Indian-Nigerian and Nigerian parents, and the diagnosis was not suspected before death.

Our screening programme was set up because of our experience with one girl with HbSS who was severely handicapped after two attacks of pneumococcal meningitis. If penicillin prophylaxis prevents one further such case we have no doubts about the cost effectiveness of screening. Now that the Department of Health and Social Security has recommended the issue of haemoglobinopathy cards awareness of sickle haemoglobin state is bound to increase, and mothers will want to know whether their children have inherited their parents' haemoglobin pattern. This, and the in creased incidence of racially mixed marriages, make it important for the screening service to be extended (preferably to all children) over the next decade. ANN WATSON

Royal Berkshire Hospital,

Reading RGI SAN

\section{Syringe exchange schemes}

Dr Gerry V Stimson and others (18 June, p 1717) discuss syringe exchange schemes for drug users. Sharing contaminated needles and syringes is likely to be the most common route of transmission of human immunodeficiency virus (HIV) among injecting drug addicts. Many drug users continue to inject, and little is known about the circumstances that lead to such high risk behaviour.

In September 1987 a questionnaire was completed by 28 injecting opiate addicts. By using this questionnaire we elicited more evidence of sharing than had been given to staff in pretreatment interviews. Ninety two per cent of subjects rated their risk of AIDS from sharing to be high or very high (four or five respectively on a five point scale). Nevertheless, $89 \%$ reported having knowingly shared a needle or syringe. Thirty six per cent of these had shared equipment the first time they injected, and $14 \%$ regularly shared equipment despite their high subjective ratings of the risk. Seventy nine per cent had shared equipment within the past 12 months-half were experiencing withdrawal at the time, of whom half experienced a sudden craving, and $20 \%$ had been unexpectedly offered drugs. Thirty six per cent had taken benzodiazepines, alcohol, or cannabis before the episode of sharing.

Of the 22 who had shared equipment in the past 12 months, $12(54 \%)$ reported that the sharing was associated with non-availability of sterile needles and syringes. Four reported that it had occurred outside chemists' opening hours, two that they did not have sufficient money, and three that the pharmacy was too far away. One did not know where to go to obtain clean equipment. Twenty two $(88 \%)$ of the 25 who had shared equipment reported using a cleaning procedure (table)

Methods used by 22 drug users to clean borrowed injection equipment before use

\begin{tabular}{lc}
\hline Method & No $(\%)$ of subjects \\
\hline Boiling water & $5(23)$ \\
Hot water & $4(18)$ \\
Bleach or Milton & $4(18)$ \\
Cold water & $3(14)$ \\
Disinfectant & $2(9)$ \\
Miscellaneous (citric acid, salt water, etc) & $4(18)$ \\
\hline
\end{tabular}

Despite awareness of the risks a large proportion of this group of drug users shared equipment. In certain circumstances the risks are forgotten or ignored. The moderate ease with which needles and syringes can be purchased from pharmacists in the London area was not sufficient to ensure the use of sterile equipment. Most subjects, however, cleaned their equipment on occasions when they shared, even though the cleaning techniques were not those recommended. The widespread use of cleaning methods suggests that the dissemination of information about more effective cleaning techniques is an important contribution to reducing the rate of spread of HIV.

JOHN STRANG SIAN HUN ANDREW JOHNS

Drug Dependence Clinical Research and Treatment Unit, Maudsley and Bethlem Royal Hospitals, London SES

Dr Gerry V Stimson and others (18 June, p 1717) make no extravagant claims about their research findings. As expected, the survey was unable to answer the central question-whether syringe exchange schemes influence a change in risk behaviour. The observation that the schemes are not successful in attracting some intravenous drug users who should be reached in a prevention strategy for human immunodeficiency virus (HIV) infection is consistent with the report by Ghodse $e$ al that a "hard core" of intravenous drug users are resistant to behaviour change.

The high drop out rate is not unexpected. The overall decline in attendance suggests that all drug misuse services, whether hospital or community based, face similar problems which cannot be resolved without enhanced resources and attractiveness of the facilities for users and care providers. ${ }^{2}$ The observed decline in attendance after the initial contact casts doubt on the epidemiological findings of those prevalence studies of drug misuse based on multiagency enumeration, from which extensive but erroneous conclusions are often drawn. A single contact with a drug misuser says nothing about where that person will be days or weeks after identification in a prevalence survey.

The authors reported that $31 \%$ of the people monitored had had no previous treatment, $35 \%$ had received treatment but were no longer in contact with the services, and $34 \%$ were receiving treatment during the survey. They failed, however, to provide any data on the differing characteristics of these three groups. The proportion of people who were subsequently given treatment is relevant but not stated. In many respects the survey has raised more questions than it answered and seems to have wider implications for drug users not receiving treatment and for retention and drop out rates than for the future of syringe exchange schemes. The suggestion that the schemes should become "low threshold" contact points or a new tier of service for drug users is not substantiated. Despite these flaws the survey provides useful data and should stimulate a constructive debate on HIV infection and services for drug misusers.

JAMES EDEH

Section of Psychiatry of Addictive Behaviour,

St George's Hospital,

London SW17

1 Ghodse AH, Tregenza G, Li M. Effect of fear of AIDS on sharing injection equipment among drug abusers. Br Med f 1987;295: 698-9.

2 Newman RG. Frustrations among professionals working in drug treatment programs. Br J Addict 1987;82:115-7.

\section{Kinesiology and food allergy}

Professor J S Garrow (4 June, p 1573) concludes that because the kinesiology response was not reproduced under the conditions of blind testing it cannot be a reliable indicator of food allergy. His conclusion rests on assumptions about the nature of kinesiology and conditions appropriate to assessing its reliability which need to be questioned if the method is not to be relegated to the dustbin without fair trial.

One assumption is that the unfamiliar method of muscle testing for food sensitivity ought to behave like the more familiar method of skin prick testing. There is no reason to make such an assumption, especially since it is not certain that the two methods are concerned with the same type of food sensitivity. There is also no reason to assume that a design which might be relevant to a study of the reliability of one method is necessarily appropriate for another.

A further questionable assumption is that the reliability of a measure may be assessed without specification of the relevant sampling frame. Determining the reliability of a measure involves drawing conclusions about its consistency or stability which may be generalised to conditions other than those in which the data providing the basis for these conclusions were generated. Intrinsic to this process is precise specification of subjects (whose response variability may be considerable) and the time intervals between tests.' The fact that neither the nature of the subjects nor the duration of the interval between tests is described in sufficient detail to permit replication limits the possibility of generalising from the particular sample and test conditions to other situations.

Another difficulty arises from the assumption that repeated testings with the same stimuli within a short interval are independent of each other. This may not be assumed: it needs to be determined empirically. In biometrics there are many examples where repeated exposures to a test within a short interval produce results that differ as a function of the prior exposure. The commonly observed reduction in blood pressure the second time an anxious patient is tested during a session 